\title{
FETAL WARFARIN SYNDROME - A CASE REPORT
}

\author{
Syed Sajid Hussain Shah $^{1}$, Bibi Aaliya ${ }^{2}$
}

\section{ABSTRACT:}

A two-month-old male infant presented with history of respiratory difficulty and got admitted with provisional diagnosis of pneumonia. On examination patient was having unilateral nasal hypoplasia and cyanosis with echocardiography showing truncus arteriosus. Detail history revealed that mother had valve replacement and she was taking warfarin during pregnancy. After initial management patient was referred to pediatric cardiac surgery and plastic surgery for further management. Parents were counseled regarding contraception and family planning.

KEYWORDS: Warfarin, Syndrome, Fetal Anomalies, Teratogenic

How to cite this Case Report:

Shah SSH, Aaliya B. Fetal Warfarin Syndrome - A Case Report. J Gandhara Med Dent Sci. 2022;9(1): 82-84

https://doi.org/10.37762/jgmds.9-1.235

Correspondence:

${ }^{1}$ Assistant Prof. Pediatric Nephrology, Institute of Kidney

Diseases, Peshawar

(v): $\quad+92-334-8951184$

凶: syed_sajid20@yahoo.com

${ }^{2}$ Assistant Prof. Pediatrics, Lady Reading Hospital, Peshawar

\section{INTRODUCTION:}

Fetal warfarin syndrome (FWS), Di Sala syndrome or warfarin embryopathy is one of the rare fetal anomalies due to exposure of fetus to warfarin in utero. ${ }^{1}$ Warfarin is a teratogenic drug that causes fetal anomaly especially in the first trimester and associated with fetal loss. ${ }^{2}$ Embryopathy due to warfarin is characterized by the abnormalities of cartilages and bones known as chondrodysplasia punctata. Babies who are affected may have nasal hypoplasia, cardiac anomalies, microphthalmia, excessive calcifications in the epiphyses and vertebrae, hypoplasia of the extremities, intrauterine growth retardation, scoliosis, deafness, and mental retardation. ${ }^{3}$ Risk of teratogenicity is not predictable with any dose of warfarin. In women who have mechanical valves and receiving warfarin, the optimal dose is not defined. ${ }^{4}$ We are reporting case of one two months old male child, diagnosed as case of FWS. After initial management patient was referred to pediatric cardiac surgery and pediatric plastic surgery for further management.

\section{CASE REPORT:}

A two-month-old male infant presented to pediatric emergency department of Ayub Teaching Hospital, Abbottabad with history of respiratory difficulty. Patient was admitted in pediatric department with provisional diagnosis of pneumonia. On clinical examination patient was having nasal hypoplasia and cyanosis. There was systolic murmur on cardiac auscultation. Echocardiography showed large membranous ventricular defect and truncus arteriosus. On detail medical history mother informed that she had cardiac surgery for sever mitral stenosis and prostatic cardiac valve was placed in heart. She has been taking warfarin for last three years. This pregnancy was spontaneous, and she only visited local doctor working as obstetrician during pregnancy in last trimester. Neither doctor inquired nor she informed about her warfarin intake. Baby was delivered via spontaneous vaginal delivery at local clinic. After birth of baby, patient was having recurrent acute respiratory infection as mother visited local doctor multiple times, but cardiac disease could not be suspected or diagnosed. Then patient got too sick to be taken to tertiary care hospital. After stabilization of patient condition patient was referred to pediatric cardiac surgery and pediatric plastic surgery for further management. Parents were counseled regarding contraception and family planning. Proper consent was taken from parents for presenting this case report. 


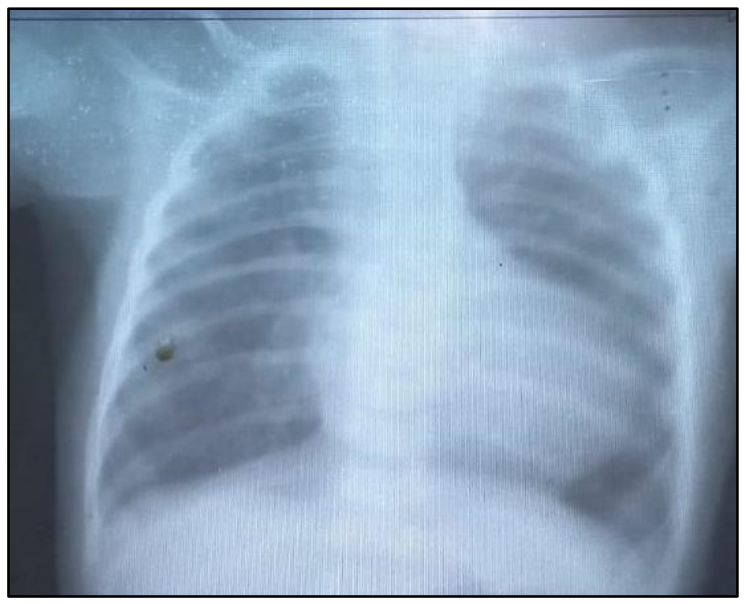

Figure 1: Patient CXR

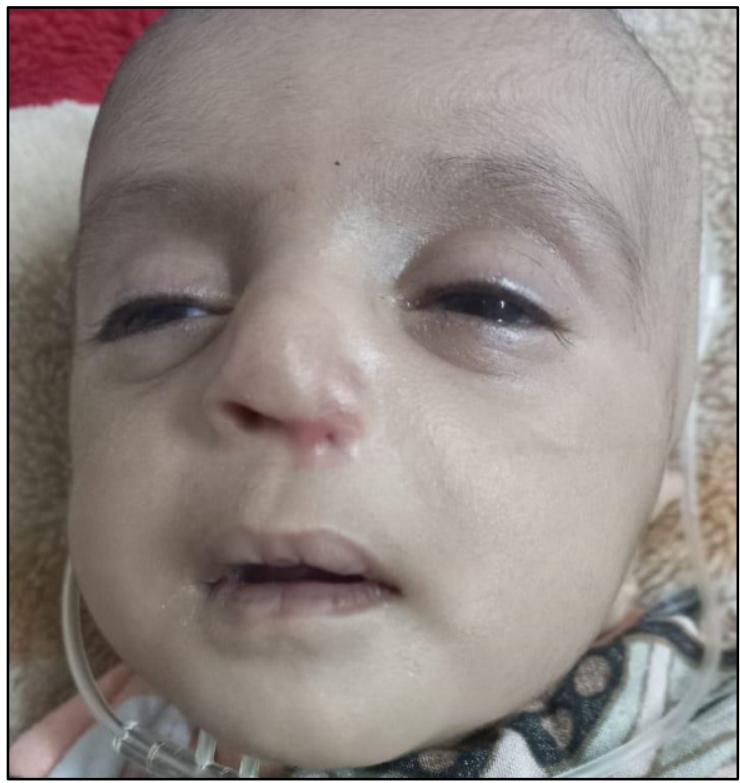

Figure 2: Patient with Nasal Hypoplasia and Cyanosis

\section{DISCUSSION:}

In developing countries including Pakistan, rheumatic heart disease is one of the major causes of cardiac illness in childbearing women. ${ }^{5}$ Cardiac diseases are there in $1-3 \%$ of pregnant women. ${ }^{6}$ Warfarin is one drug of choice for the women with valve replacement due to RHD but there is risk of fetal malformation and abortion. ${ }^{3}$ Di Sala in 1966, reported one neonate born to mother having mitral valve prosthesis and she was taking warfarin during first trimester of pregnancy. Baby had clinical features of nasal structure hypoplasia, bilateral optic atrophy and blindness, and later on mental retardation. Then Holmes et al mentioned same infant and illustrated as a warfarin embryopathy. ${ }^{7}$ In our case mother kept on taking warfarin during first trimester of pregnancy and baby had nasal hypoplasia and cardiac anomaly. Warfarin also causes chondrodysplasia punctata, which is abnormal calcification in the cartilage of developing bones. ${ }^{8}$ But x-ray long bones in our patient did not reveal the finding of chondrodysplasia punctata. Unfractionated heparin or low molecular weight heparin are used especially during first trimester of pregnancy instead of oral anticoagulants as still there is no consensus on use of anticoagulants in pregnancy. ${ }^{9,10}$ In our case, the pregnancy was spontaneous. Mother also did not visit during the first trimester of pregnancy and after delivery there was no referral for baby by the doctor despite having nasal hypoplasia. There should be standard protocols implemented including family planning and contraception for mothers with reproductive age who are started on teratogenic drugs. ${ }^{11}$ The purpose of this case report is to emphasize that warfarin exposure during pregnancy especially during the first trimester can lead to fetal defects and congenital anomalies. Proper history and counselling should be part of management of mothers having RHD and valve replacement taking oral anticoagulants.

\section{CONCLUSION:}

It was concluded that warfarin causes fetal defects and congenital anomalies as in this case the child had unilateral nasal hypoplasia and cyanosis with echocardiography showing truncus arteriosus

\section{CONFLICT OF INTEREST: None}

FUNDING SOURCES: None

\section{REFERENCES:}

1. Sousa AR, Barreira R, Santos E. Low-dose warfarin maternal anticoagulation and fetal warfarin syndrome. BMJ Case Reports. 2018;2018:bcr-2017-223159.

2. Güner A, Kalçık M, Gürsoy MO, Gündüz S, Astarcioğlu MA, Bayam $\mathrm{E}$, et al. Comparison of different anticoagulation regimens regarding maternal and fetal outcomes in pregnant patients with mechanical prosthetic heart valves (from the Multicenter ANATOLIA-PREG Registry). Am J Cardiol. 2020;127:113-9. https://doi.org/10.1016/j.amjcard.2020.04.010 
3. Ali CA, Varghese TC, Sreenivasan VK. A preventable syndrome for a precious baby. Int J Orthop Sci. 2021;7(1):1022-3. https://doi.org/10.22271/ortho.2021.v7.i1o.2 602

4. Dhillon SK, Edwards J, Wilkie J, Bungard TJ. High-versus low-dose warfarin-related teratogenicity: a case report and systematic review. J Obstet Gynaecol Can. 2018;40(10):1348-57.

https://doi.org/10.1016/j.jogc.2017.11.020

5. Watkins DA, Johnson CO, Colquhoun SM, Karthikeyan G, Beaton A, Bukhman G, et al. Global, regional, and national burden of rheumatic heart disease, 1990-2015. N Engl J Med. 2017;(37):713-22.

6. Remenyi B, El-Guindy A, Smith Jr SC, Yacoub M, David R Holmes Jr DR. Valvular aspects of rheumatic heart disease. Lancet. 2016;387(10025):1335-46. doi.org/10.1016/S0140-6736(16)00547-X
7. Alrukban H, Chitayat D. Fetal chondrodysplasia punctata associated with maternal autoimmune diseases: a review. Appl Clin Genet. 2018;11:31-44.

8. Songmen S, Panta OB, Paudel S, Ghimire RK. Chondrodysplasia punctata: a case report of fetal warfarin syndrome. J Nepal Health Res Counc. 2017;15(35):81-4.

9. Panduranga $\mathrm{P}$, El-Deeb $\mathrm{M}$, Jha $\mathrm{C}$. Mechanical prosthetic valves and pregnancy: a therapeutic dilemma of anticoagulation. Sultan Qaboos Univ Med J. 2014;14:e448-54.

10. Singh A, Panghal A, Prasad R, Mishra OP. A case of coumarin embryopathy after in utero exposure to acenocoumarol. J Fetal Med. 2018;5:233-6. https://doi.org/10.1007/s40556-018-0182-2

11. Conradie M, Henderson BD, van Wyk C. Preventable warfarin-induced birth defects: a missed opportunity?. S Afr Med J. 2019;109(6):415-20.

\section{CONTRIBUTORS}

1. Syed Sajid Hussain Shah - Concept \& Design; Data Analysis/Interpretation; Drafting Manuscript

2. Bibi Aaliya - Data Acquisition; Critical Revision

LICENSE: JGMDS publishes its articles under a Creative Commons Attribution Non-Commercial Share-Alike license (CC-BY-NC-SA 4.0) COPYRIGHTS: Authors retain the rights without any restrictions to freely download, print, share and disseminate the article for any lawful purpose. It includes scholarly networks such as Research Gate, Google Scholar, LinkedIn, Academia.edu, Twitter, and other academic or professional networking sites. 